\title{
Article \\ Overexpression of Transcription Factor GmTGA15 Enhances Drought Tolerance in Transgenic Soybean Hairy Roots and Arabidopsis Plants
}

\author{
Zhanyu Chen ${ }^{1}$, Xiaokun Fang ${ }^{1}$, Xueshun Yuan ${ }^{2}$, Yingying Zhang ${ }^{2}$, Huiying Li ${ }^{2}$, Ying Zhou ${ }^{2}$ and Xiyan Cui ${ }^{2, *(D)}$ \\ 1 College of Agronomy, Jilin Agricultural University, Changchun 130118, China; \\ chenzhanyu2020@163.com (Z.C.); fangxk14@163.com (X.F.) \\ 2 College of Life Sciences, Jilin Agricultural University, Changchun 130118, China; \\ yuanxueshunyxs@163.com (X.Y.); yingyingzhangyyz@163.com (Y.Z.); lihuiyingjlau@163.com (H.L.); \\ zhouying345@126.com (Y.Z.) \\ * Correspondence: cuixiyan@jlau.edu.cn; Tel.: +86-431-8453-3182
}

Citation: Chen, Z.; Fang, X.; Yuan, X.; Zhang, Y.; Li, H.; Zhou, Y.; Cui, X. Overexpression of Transcription Factor GmTGA15 Enhances Drought Tolerance in Transgenic Soybean Hairy Roots and Arabidopsis Plants. Agronomy 2021, 11, 170. https:// doi.org/10.3390/agronomy11010170

Received: 25 November 2020

Accepted: 15 January 2021

Published: 18 January 2021

Publisher's Note: MDPI stays neutral with regard to jurisdictional claims in published maps and institutional affiliations.

Copyright: (c) 2021 by the authors. Licensee MDPI, Basel, Switzerland. This article is an open access article distributed under the terms and conditions of the Creative Commons Attribution (CC BY) license (https:// creativecommons.org/licenses/by/ $4.0 /)$.

\begin{abstract}
Soybean (Glycine max) is one of the important oil crops worldwide. In recent years, environmental stresses such as drought and soil salinization have severely deteriorated soybean yield and quality. We investigated the overexpression of the transcription factor GmTGA15 in response to drought stress in transgenic soybean hairy roots and Arabidopsis plants. The results of quantitative real time polymerase chain reaction (qRT-PCR) analyses showed that GmTGA15 was greatly induced by salt, PEG6000, salicylic acid (SA), gibberellic acid (GA), abscisic acid (ABA), and methyl jasmonate (MeJA) in soybean. In response to drought stress, the contents of both chlorophyll and proline were significantly increased, while the content of malondialdehyde (MDA) was significantly decreased in the soybean hairy roots with the overexpression of GmTGA15 in comparison to wild type (WT). Under the simulated drought conditions, the transgenic Arabidopsis plants showed significantly longer roots and lower mortality than that of the wild type. These results suggest that GmTGA15 promotes tolerance to drought stress in both soybean and Arabidopsis plants. This study provides the scientific evidence for further functional analysis of soybean TGA transcription factors in drought stress and the breeding of drought-resistance crops.
\end{abstract}

Keywords: soybean hairy roots; Arabidopsis; TGA transcription factor; abiotic stress; drought tolerance

\section{Introduction}

Plants often face abiotic and biological stresses such as drought, poisoning, salinealkali, and pathogenic bacteria, which severely affect the growth and yield of crops. In longterm evolution, plants are induced to maintain their own growth and development through stress-responsive genes. These genes are divided into two categories, one containing the cell metabolism genes and stress-resistance genes, and the other the genes encoding the transcription factors, protein kinases, and phosphatases [1,2]. Transcription factors regulate the expression of genes by specifically binding to cis-acting elements on the promoter, while a single transcription factor can simultaneously bind to the promoters of different target genes.

The TGACG motif-binding factor (TGA) is a transcription factor belonging to the subfamily D of the basic leucine zipper (bZIP) family, and specifically binds to the activation sequence 1 (as-1) with the core sequence of TGACGT [3]. Transcription factors play vital roles in plant stress resistance and growth by regulating the transcription level of target genes. For example, Katagiri et al. used the cauliflower mosaic virus (CaWV) 35S promoter to identify the first TGA gene (i.e., the transcription factor TGA1a gene) in tobacco and determined its recognition of TGACGT-specific sequences [4]. To date, TGA genes have been isolated and identified in many crop and model plants including Arabidopsis, rice, wheat, Begonia, persimmon, cotton, and papaya [5-10]. 
Each TGA transcription factor contains a zinc finger domain and the glutamine transcription activation domain. The zinc finger domain is an alpha helix-rotation-alpha helix structure with a dimer consisting of a leucine zipper region and a basic region located at the C-terminus, while the glutamine activation domain is an acidic transcription activation domain [11]. The TGA transcription factors recognize the cis-acting element TGACGT with its core sequence of ACGT bound to the basic domain, while the leucine zipper region participates in oligomerization. The $\mathrm{N}$-terminus of the transcription factor contains the acidic activation region STDxDT forming a dimer with the alkaline region binding directly to DNA [11].

Studies have shown that TGA transcription factors are mainly involved in the processes of plant growth and development, resistance to diseases, and stress regulation. For example, members of TGA transcription factors often interact with the regulatory factors NPR1, GRX480, ERF72, ARR2, and SCL14, participating in various disease-resistance or stress-resistance signal transduction pathways, such as salicylic acid (SA), jasmonic acid (JA), and abscisic acid (ABA), ultimately enhancing the tolerance of plants to adverse stresses [12]. In Arabidopsis, both the TGA1 and TGA4 regulate not only the biosynthesis of SA and pipecolic acid (PA) by regulating the expression of genes SARD1 and CBP60g [13] but also the root response to nitrate [14]. Specifically, the nitrate signal transmitted by TGA1/TGA4 directly regulates the CAPRICE (CPC) root hair cell fate determination genes to increase root hair formation in Arabidopsis [15]. Studies have shown that AtTGA4 enhances drought resistance by increasing the nitrate transport and assimilation in Arabidopsis [16], while TGA7 may respond to plant drought stress by negatively regulating the expression of downstream gene AtBGI [17]. In soybean, three genes (i.e., GmbZIP44, GmbZIP62, and GmbZIP78) have demonstrated the ability to enhance salt tolerance and freezing tolerance of transgenic Arabidopsis as negative regulators of ABA signal transduction [18], while the bZIP transcription factor MdbZIP44 in apple regulates ABA to promote anthocyanin accumulation [19]. Furthermore, the overexpression of GmFDL19 enhances the drought and salt tolerance of soybean [20], while the nuclear protein GmbZIP110 shows transcriptional activation activities and plays an important role in response to salt stress in soybean [21]. Recently, studies have shown that GmTGAs play important roles in symbiotic nodulation and response to nitrogen utilization in soybean [22].

To date, a total of 27 GmTGA genes have been identified in the soybean genome with two of them (i.e., GmTGA15 and GmTGA17) up-regulated by 10 and 17 times, respectively, in the soybean seedlings under drought-stress treatment [23]. Furthermore, it has been demonstrated that the overexpression of GmTGA17 enhances both drought tolerance and salt tolerance in transgenic Arabidopsis and soybean hairy roots [23]. Although both GmTGA15 and GmTGA17 share a lot of molecular similarities, the variations between these two transcription factors warrant further studies to verify and characterize the functions of these and many other transcription factors identified previously [23]. Our previous studies have shown that GmTGA15 is a nuclear protein in soybean containing a highly conserved bZIP domain and a DOG1 structure and showing the characteristics of transcriptional activation [24]. Due to these shared molecular structures and characteristics with GmTGA17, it is speculated that GmTGA15 could enhance tolerance to both drought and salt stresses in crop plants. To date, the functional studies of GmTGA15 in crop plants under environmental stresses are sparse.

In our study, we investigated the molecular functions of the overexpression of GmTGA15 in response to drought stress in transgenic soybean hairy roots and Arabidopsis plants. Our results showed that GmTGA15 significantly enhances drought tolerance in both soybean hairy roots and transgenic Arabidopsis plants. This study provides valuable information for the further functional analysis of soybean TGA transcription factors in drought stress and the breeding of drought-resistance crops. 


\section{Materials and Methods}

\subsection{Plant Materials, Growth Conditions, and Treatments}

Plants of both soybean (Glycine max cultivar Williams 82) and Arabidopsis thaliana were grown in a mixture of nutrient soil and vermiculite (3:1) inside the growth chamber with a humidity of $50 \%$ under a daily light illumination $\left(300 \mu \mathrm{mol} \cdot \mathrm{m}^{-2} \cdot \mathrm{s}^{-1}\right.$ for soybean and $120 \mu \mathrm{mol} \cdot \mathrm{m}^{-2} \cdot \mathrm{s}^{-1}$ for Arabidopsis) of $14 \mathrm{~h}\left(7 \mathrm{a} . \mathrm{m}\right.$. to $9 \mathrm{p} . \mathrm{m}$.) at $25^{\circ} \mathrm{C}$, then dark for $10 \mathrm{~h}\left(9 \mathrm{p} . \mathrm{m}\right.$. to $7 \mathrm{a}$ a.m.) at $22^{\circ} \mathrm{C}$. The young roots of soybean plants with the first 3-leaflet compound leaf unfolded were selected as the experimental materials.

\subsection{Plasmid Construction and Agrobacterium Rhizogenes-Mediated Transformation of Soybean Hairy Roots}

In our previous study [24], the GmTGA15 gene, with a length of 1089 base pairs containing eight exons and seven introns, was cloned from soybean plants of the cultivar Williams 82. Our results showed that the protein GmTGA15 was composed of 362 amino acids with a molecular weight of $41.01 \mathrm{kDa}$ belonging to the family of bZIP transcription factors and was located in the nucleus, showing evident transcriptional activation activities. The full-length coding sequence of GmTGA15 amplified was inserted into the BamH I site of a pCAMBIA3301 vector using the forward primer (5'TCCAGCTCCAGGATCCATGAATTCAGCATCCCCGCA-3 $\left.3^{\prime}\right)$ and the reverse primer $\left(5^{\prime}-\right.$ GAGAAAGCTTGGATCCCTAAGCAGGTTCCCGGGGTCTAT-3'). The pCAMBIA3301GmTGA15 plasmid was then transformed into soybean hairy roots by the high-efficiency Agrobacterium rhizogenes-mediated method, as described previously [25].

\subsection{Expression Analysis by $q R T-P C R$}

For expression analysis, seedlings of soybean were exposed to a net floating on the surface of a solution containing 20\% PEG6000, $200 \mathrm{mM} \mathrm{NaCl}, 100 \mu \mathrm{M}$ abscisic acid (ABA), $200 \mu \mathrm{M}$ gibberellic acid (GA), $2 \mathrm{mM}$ salicylic acid (SA), and $50 \mu \mathrm{M}$ Methyl jasmonate (MeJA), respectively. The selection of these four types of phytohormones as indicators of drought stress was based on previous studies [26,27]. The plant materials were sampled at $0,1,3,6,12$, and $24 \mathrm{~h}$, respectively, immediately frozen in liquid nitrogen, and kept in storage at $-80^{\circ} \mathrm{C}$. Total RNA was extracted from the seedlings of soybean using the plant total RNA kit (TaKaRa, Shiga, Japan). To study the relative expression patterns of GmTGA in soybean, total RNA samples were also extracted from the different tissues or organs (i.e., roots, stems, leaves, flowers, pods, and seeds) at four different developmental stages in soybean, including the seedling stage (without branches on the stems), branching stage (with branches on the stems), flowering stage (with the presence of flowers), and podding stage (with the presence of pods and seeds). Samples were generally collected in the morning and were immediately frozen in liquid nitrogen, then kept in storage at $-80^{\circ} \mathrm{C}$. The reverse transcription for the first strand cDNA synthesis for each plant sample was performed with $2 \mu \mathrm{g}$ of total RNA using the first-strand DNA synthesis kit (TaKaRa, Shiga, Japan). The qRT-PCR analyses were performed on a MX3000P Real-Time system containing 12.5- $\mu \mathrm{L} 2 \times$ SYBR Premix Ex Taq (TaKaRa, Shiga, Japan) and 9.5- $\mu$ L double distilled water using the GmTGA15 specific forward primer 5'-TGGACACTGGGTGAATGAGC-3' and reverse primer $5^{\prime}$-GTCAGGGGTTCAATCAGGGG- $3^{\prime}$. The procedure to conduct the melting curve analysis was as follows: 1 cycle for $30 \mathrm{~s}$ at $95^{\circ} \mathrm{C}$, followed by 30 cycles for $5 \mathrm{~s}$ at $95^{\circ} \mathrm{C}$ and $20 \mathrm{~s}$ at $60^{\circ} \mathrm{C}$, and 1 cycle for $60 \mathrm{~s}$ at $95^{\circ} \mathrm{C}, 30 \mathrm{~s}$ at $55^{\circ} \mathrm{C}$, and $30 \mathrm{~s}$ at $95^{\circ} \mathrm{C}$. The inner reference gene EF1- $\alpha$ (forward primer $5^{\prime}$-TGCAAAGGAGGCTGCTAACT- $3^{\prime}$ and reverse primer $5^{\prime}$-CAGCATCACCGTTCTTCAAA- $3^{\prime}$ ) was used to normalize the data. The quantitative variations of gene expression between the examined replicates were evaluated by the $2^{-\Delta \Delta C t}$ method as described previously [28]. Three independent biological repeats were performed to ensure accurate statistical analysis. 


\subsection{Drought Treatment in Transgenic Soybean Hairy Roots}

The transgenic soybean hairy roots with the overexpression of GmTGA15 and the control group were selected to conduct the abiotic stress tolerance assays. Recombinant vectors pCAMBIA3301-GmTGA15 were transformed into the soybean hairy roots by Agrobacterium rhizogenes K599-mediated transformation [25]. The transgenic soybean hairy roots were then treated with 10\% (w/v) PEG6000 solution for 1 week, while the control group continued to grow under normal conditions without the treatment of PEG6000. As the three indicators of drought stress, the contents of malondialdehyde (MDA), chlorophyll, and proline in leaves were measured to evaluate the drought tolerance of the soybean plants [29].

\subsection{Generation of Transgenic Arabidopsis Plants}

The function of enhancing drought tolerance by GmTGA15 was further evaluated in transgenic Arabidopsis plants with the heterologous overexpression of GmTGA15. The CDS sequence of GmTGA15 was amplified and cloned into the pCAMBIA3301 vector. The recombinant expression vector containing pCAMBIA3301-GmTGA15 was transformed into the Agrobacterium tumefaciens strain Agl0 and subsequently transformed into Arabidopsis ecotype Col-4 by the floral dip method [30]. The harvested seeds were screened by Basta tolerance, while the T3-transformed plants were confirmed by qRT-PCR analysis and used for further study based on the expression level of GmTGA15. Three independent transgenic lines of Arabidopsis were used to evaluate their drought tolerance. For the root growth analysis, 5-day-old seedlings initially grown in 1/2 MS medium were transferred to $1 / 2$ MS medium containing 6\% ( $\mathrm{m} / \mathrm{v})$ PEG6000 for vertical growth under a photoperiod of $16-\mathrm{h}$ with light of $120 \mu \mathrm{mol} \cdot \mathrm{m}^{-2} \cdot \mathrm{s}^{-1}$ and $8 \mathrm{~h}$ in dark at $22^{\circ} \mathrm{C}$. The lengths of roots were measured 1 week before and after the treatment of $6 \%$ PEG6000. The relative root elongation (RRE) was calculated as (root elongation in drought treatment/root elongation in control) $\times 100$. For the growth under the drought stress in soil, 2-week-old seedlings were not watered until phenotypic differences on leaves were observed. Then, plants were watered and recovered for 1 week to calculate the survival rates under the drought-stress treatment.

\subsection{Measurement of Chlorophyll, Proline, and Malondialdehyde (MDA)}

The content of free proline in plants was determined following the methods as described previously with slight modifications [31]. Briefly, $0.2 \mathrm{~g}$ of plant sample were homogenized in $7 \mathrm{~mL}$ of $3 \%$ sulfosalicylic acid using a mortar. The 2 -mL supernatant was transferred to a new tube with the extraction solution containing $4 \mathrm{~mL}$ of acid ninhydrin, $2 \mathrm{~mL}$ sulfosalicylic acid, and $2 \mathrm{~mL}$ of glacial acetic acid. The reaction mixture was boiled in a water bath at $100{ }^{\circ} \mathrm{C}$ for $1 \mathrm{~h}$ then stored at $4{ }^{\circ} \mathrm{C}$ for $30 \mathrm{~min}$. Then, a total of $4 \mathrm{~mL}$ of toluene was added to the solution and shaken to extract the upper red product. The proline content for each sample was measured based on the absorbance at $520 \mathrm{~nm}$. The content of MDA in plants was determined following the methods as described previously with minor modifications [32]. Specifically, $0.1 \mathrm{~g}$ leaf sample with $1 \mathrm{~mL}$ of $0.05 \mathrm{M}$ phosphate buffer ( $\mathrm{pH}$ 7.8) and a small amount of quartz sand added in a mortar was ground into a homogenate, which was poured into a 15-mL centrifuge tube, then the mortar was washed with $4 \mathrm{~mL}$ of phosphate buffer with the solution transferred to the centrifuge tube for centrifugation at $10,000 \times g$ and $4{ }^{\circ} \mathrm{C}$ for $20 \mathrm{~min}$. The content of chlorophyll in plants was determined following the methods as described previously with slight modifications [33]. Briefly, $0.1 \mathrm{~g}$ soybean leaves were cut into 1-mm long sections with a razor blade then completely submerged under $2 \mathrm{~mL}$ of dimethyl sulfoxide for extraction of chlorophyll in a $65{ }^{\circ} \mathrm{C}$ water bath in the dark until leaves became whitish or transparent. Then, $8 \mathrm{~mL}$ $80 \%$ acetone was added to the cooled extraction solution. The chlorophyll content for each sample was measured based on absorbance at $520 \mathrm{~nm}$. All experiments were repeated with three replicates. 


\subsection{Data Analysis}

All data were presented as the mean of three biological replicates \pm standard deviation (SD). One-way ANOVA tests using DPS 11.0 edition for windows were conducted to identify the significant difference between the treatments, with the $p$ values set to 0.01 or 0.05 [34].

\section{Results}

\subsection{Expression Patterns of GmTGA15 under Abiotic Stresses and Hormone Treatments}

To investigate the expression patterns of GmTGA15 in response to abiotic stresses and hormone treatments, quantitative real-time PCR (qRT-PCR) was performed to analyze the transcript abundance of GmTGA15 in soybean plants of cultivar Williams 82 (Figure 1). To further analyze the expression patterns of GmTGA genes, the transcript levels of GmTGA15 were analyzed in six different tissues and organs of soybean plants at four different developmental stages (Figure 2).

Various expression patterns of GmGAT15 in soybean hairy roots were observed under different abiotic stresses and hormone treatments. GmTGA15 was quickly induced by the treatment of $200 \mathrm{mM} \mathrm{NaCl}$ with the total RNA of GmTG15, increasing up to the highest level of $\sim 3.3$-fold over $3 \mathrm{~h}$ in roots (in comparison to that of the control) before starting to gradually decline for the rest of the experiments over $24 \mathrm{~h}$ (Figure 1A). Under the treatment by PEG6000, the expression level of GmTGA15 gene showed an overall upward trend, increasing up to the highest level of $\sim 7.2$-fold over $24 \mathrm{~h}$ in roots compared to the control (Figure 1B). Similar to the treatment of $\mathrm{NaCl}$, under the treatment of $2 \mathrm{mM} \mathrm{SA}$, the expression of GmTGA15 increased up to the highest level of $\sim 5.1$-fold over $3 \mathrm{~h}$ in comparison to that of the control, then declined until the end of the experiment (Figure $1 \mathrm{C}$ ). The treatments of both 100- $\mu \mathrm{M} \mathrm{ABA}$ (Figure 1D) and 50- $\mu \mathrm{M}$ MeJA (Figure 1E) stresses increased the expression levels of GmTGA15 in roots $\sim 2$-fold in comparison to that of the control groups over $24 \mathrm{~h}$, with varied levels of expression prior to the end of the experiments. Under the treatment of $200 \mu \mathrm{M} \mathrm{GA}$, the expression of GmTGA15 increased quickly up to the highest level of $\sim 3$.8-fold over $1 \mathrm{~h}$ compared to that of the control, then started to gradually decline to the level comparable with that of the control as it reached 24 h (Figure 1F).
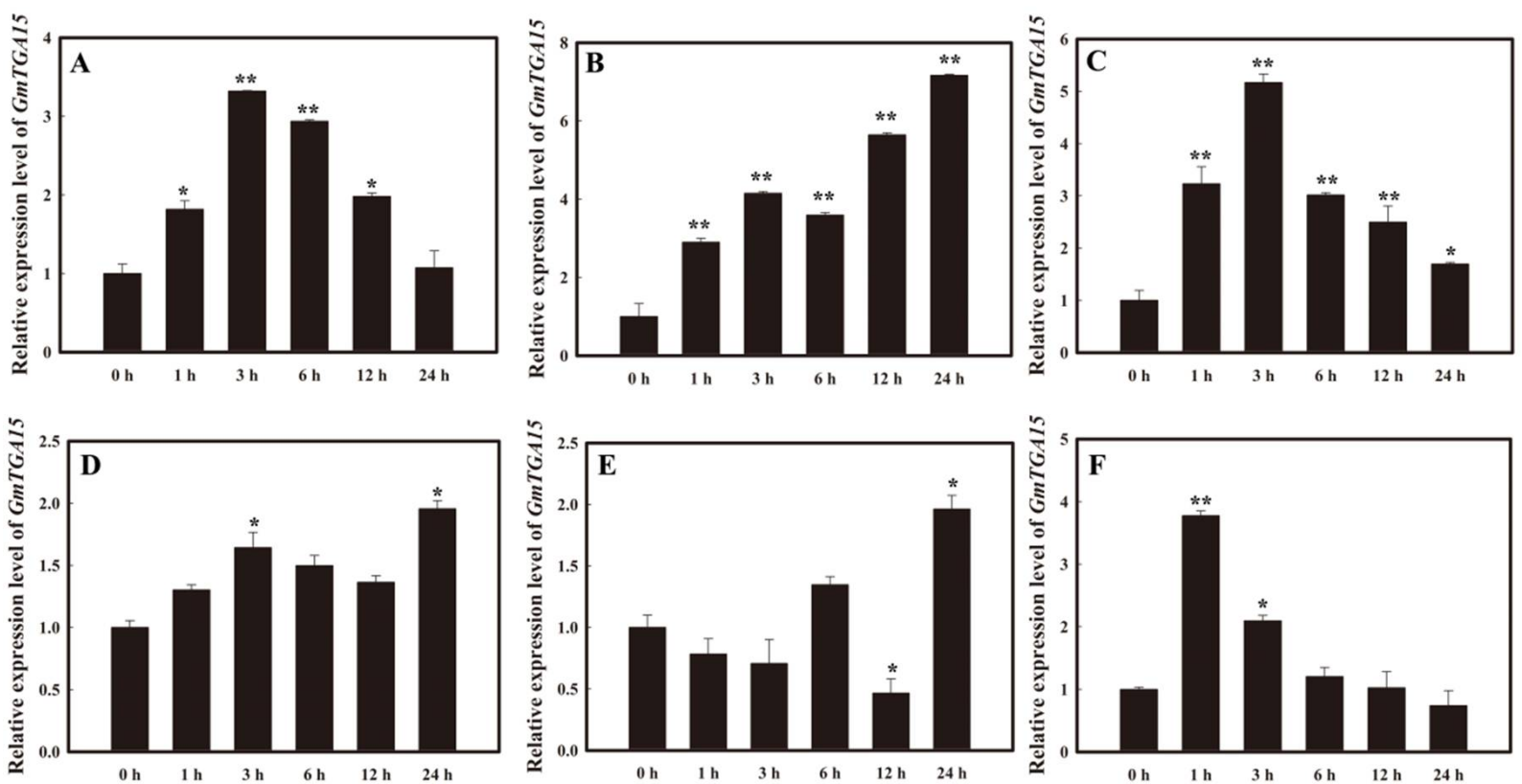

Figure 1. The relative expression of gene GmTGA15 under abiotic treatments of $\mathrm{NaCl}(\mathbf{A})$ and PEG6000 (B) and hormone treatments of SA (C), ABA (D), MeJA (E), and GA (F) in soybean hairy roots based on qRT-PCR analysis. Symbols * and ** indicate significant differences at $p$ values of 0.05 and 0.01 , respectively. 

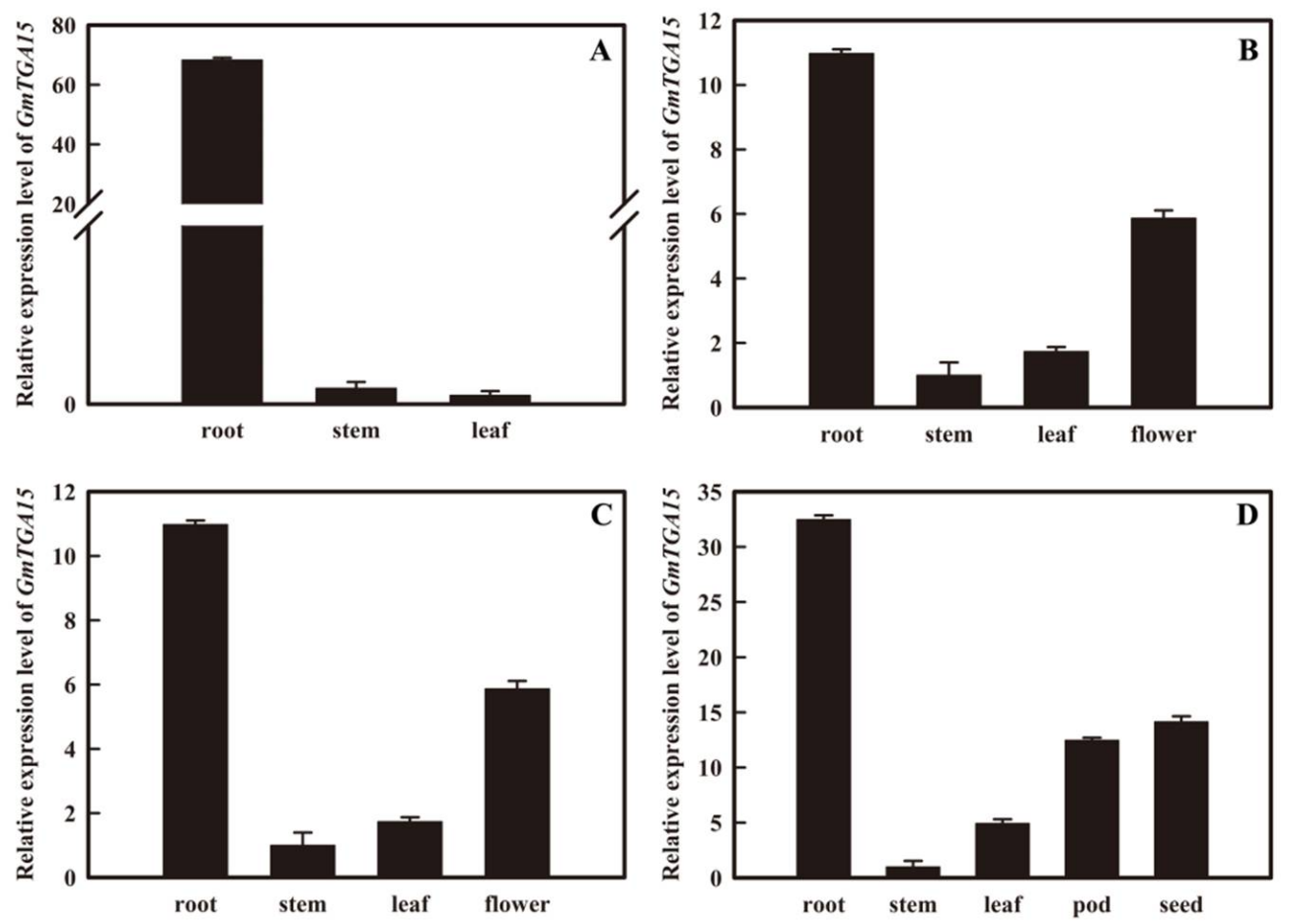

Figure 2. The relative expression of GmTGA15 in different tissues or organs of soybean plants at seedling (A), branching (B), flowering (C), and podding (D) stages based on qRT-PCR analysis.

The expression patterns of GmTGA15 were investigated in different tissues or organs of the soybean plants, including the root, stem, leaf, flower, pod, and seed samples, at four different developmental stages. The results of the qRT-PCR analysis showed that the expression levels in different tissues or organs during different developmental stages varied greatly, indicating that GmTGA15 was expressed constitutively with different functions in different tissues or organs during different developmental stages in soybean (Figure 2). Among the four developmental stages, GmTGA showed the highest expression level during the seedling stage, followed by the podding stage (Figure 2). In the seedling stage, roots showed the highest expression level among all examined organs, namely, 68 -fold higher than those in either stems or leaves (Figure 2A). In the branching stage, the expression levels in both stems and leaves were similar and $\sim 3$-fold lower than that of roots (Figure $2 \mathrm{~B}$ ), while the expression level in flowers was $\sim 6$-fold higher than those in either stems or leaves in the flowering stage (Figure 2C). In the podding stage, the expression levels in pods and seeds were $\sim 12$-fold and $\sim 14$-fold higher than that of stems, respectively, while the roots showed the highest expression level at $\sim 32$-fold that of the stems (Figure 2D).

\subsection{GmTGA15 Improves Tolerance to Drought Stress in Transgenic Soybean Hairy Roots}

The successful construction of the plant expression vector pCAMBIA3301-GmTGA15 and its transformation into soybean hairy roots was confirmed by PCR (Figure S1). The functions of GmTGA15 under drought stress were further investigated by performing abiotic stress assays induced by PEG6000 in Agrobacterium rhizogenes-mediated soybean hairy roots. The successful amplifications of $35 \mathrm{~S}$ promoter and the bar gene located on the vector pCAMBIA3301 were observed in the GmTGA15 transgenic soybean hairy roots but not in the Agrobacterium rhizogenes-transformed soybean hairy roots (Figure 3).

The overexpression of GmTGA15 was evaluated by the qRT-PCR analysis, revealing that the expression level of GmTGA15 was significantly higher (i.e., overexpressed) in three lines of soybean hairy roots with the overexpression of GmTGA15 by more than 4 -fold compared to the transgenic Agrobacterium hairy roots (Figure 4). Due to their significantly increased expression levels of GmTGA15, these three lines of soybean plants were selected for further treatments of drought stress in order to study the change of proline, chlorophyll, 
and MDA. The results showed that in all three lines, under the treatment of drought stress induced by $20 \%$ PEG6000, the contents of both proline and chlorophyll were significantly increased, while the MDA was significantly reduced in soybean hairy roots with the overexpression of GmTGA15 compared with the control (Figure 5).

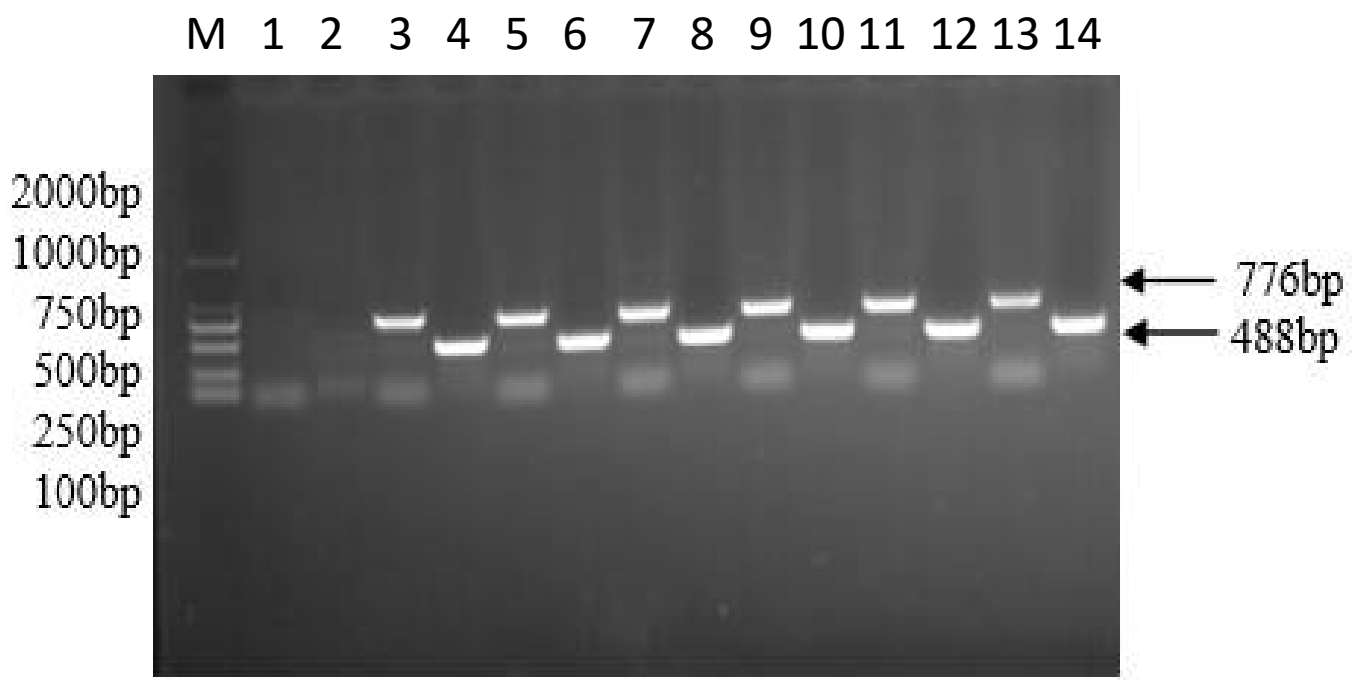

Figure 3. Construction of soybean plant expression vector pCAMBIA3301-GmTGA15 in transgenic GmTGA15 soybean hairy roots. Lane M indicates the DL2000 markers. Lanes 1 and 2 contain the PCR-amplified products of $35 \mathrm{~S}$ promoter and bar gene in the transgenic Agrobacterium hairy roots (no band). Lane pairs 3 and 4, 5 and 6, 7 and 8,9 and 10, 11 and 12, and 13 and 14 show the PCR-amplified products of $35 \mathrm{~S}$ promoter $(776 \mathrm{bp})$ and the bar gene $(488 \mathrm{bp})$ of six lines of randomly chosen transgenic GmTGA15 soybean hairy roots.

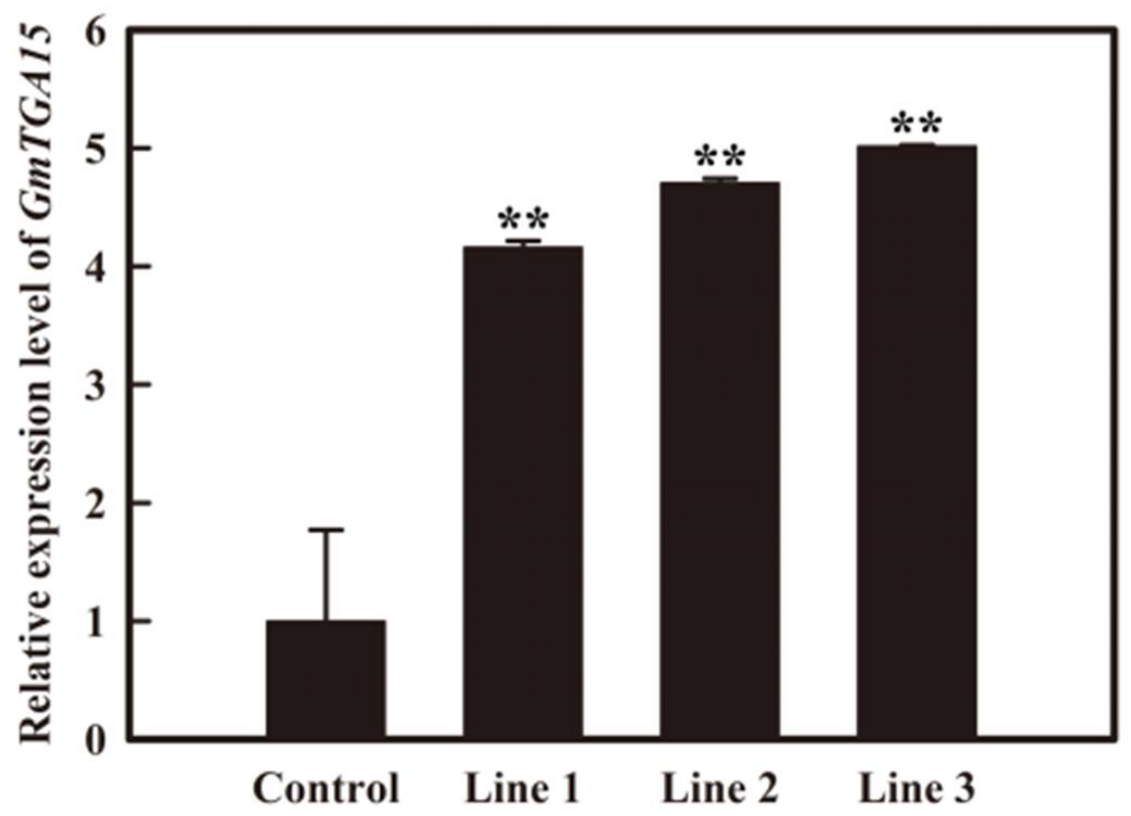

Figure 4. The relative expression of transgenic GmTGA15 soybean in three randomly chosen lines. Control contains the transgenic Agrobacterium lines. Symbols ${ }^{* *}$ indicate a significant difference at $p$ values of 0.01 . 

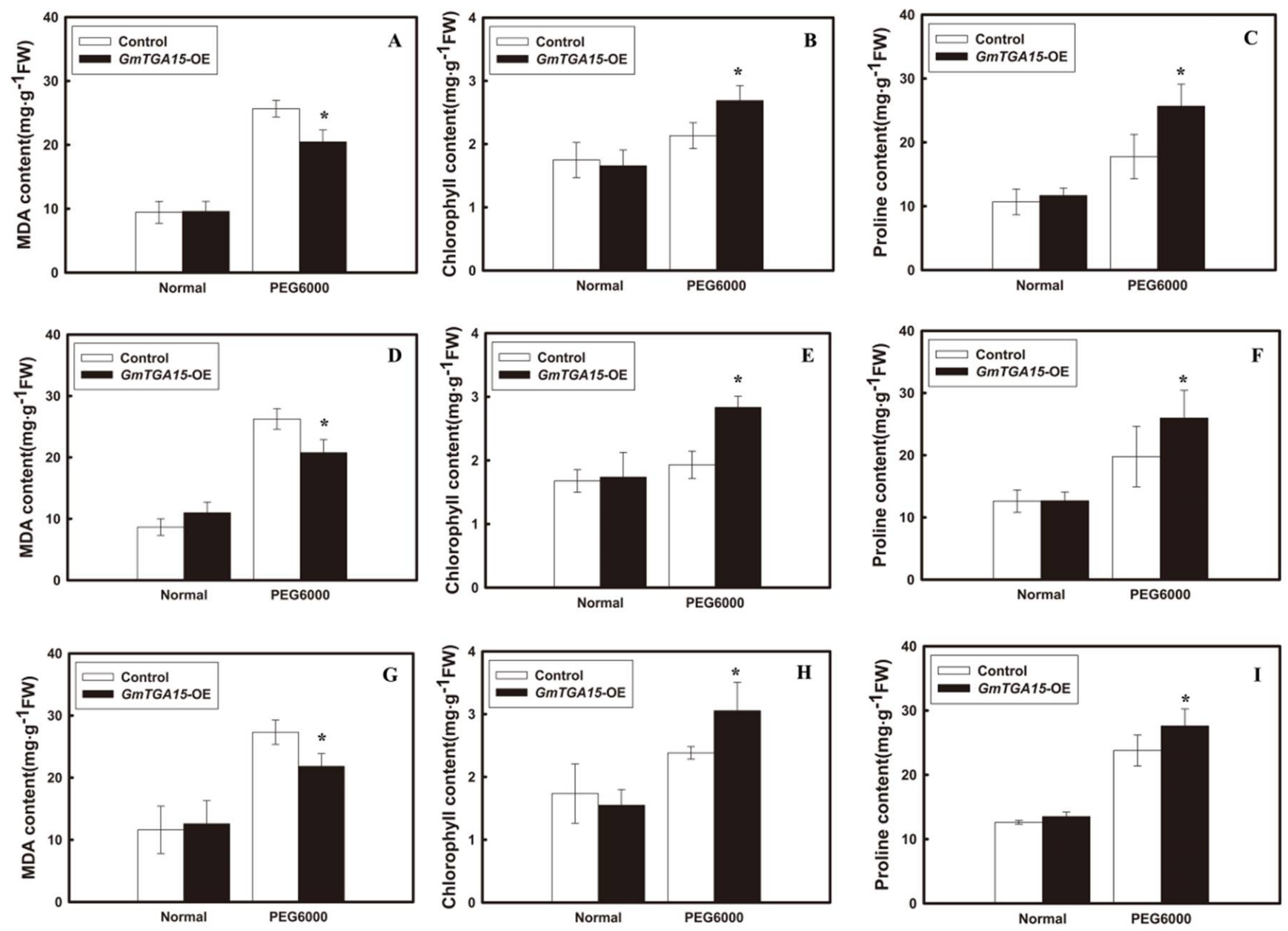

Figure 5. The contents of MDA, chlorophyll, and proline of three lines (Lines 1-3) of soybean hairy roots with the overexpression of GmTGA15 induced by the treatment of 20\% PEG6000 for 7 days. The contents of MDA, chlorophyll, and proline of Lines 1, 2, and 3 (Figure 4) are presented in (A-C), (D-F), and (G-I), respectively. The soybean hairy roots labelled as "normal" were not treated with PEG6000. Control indicates the transgenic Agrobacterium lines, while the GmTGA15-OE presents the transgenic GmTGA15 lines with the overexpression of GmTGA15. Symbol * indicates the significant difference between treatments at $p$ values of 0.01 .

\subsection{Overexpression of GmTGA15 Improved the Drought Tolerance in Transgenic Arabidopsis Plants}

Having demonstrated the function of enhancing drought tolerance in soybean, GmTGA15 was then overexpressed in Arabidopsis plants grown in both medium (containing 6\% PEG6000 simulating drought-stress conditions) and soil to further confirm its roles in drought tolerance. Both the T3 transgenic Arabidopsis lines and wild type (WT) were sown in $1 / 2$ MS medium with and without $6 \%$ PEG6000, respectively. In the absence of $6 \%$ PEG6000, the total root length and survival rates showed no significant difference between transgenic and WT plants of Arabidopsis (Figure 6A,B). In contrast, when exposed to 1/2 MS medium with 6\% PEG6000 for 8 days, the growth of transgenic and WT plants was strongly inhibited, while the transgenic Arabidopsis plants showed significantly longer root lengths than those of the WT plants. Under drought-stress conditions, the WT plants either shriveled severely or died in two weeks, while the transgenic Arabidopsis plants just started to shrivel slightly and showed significantly lower mortality than that of the WT plants (Figure 6B,C). 
A

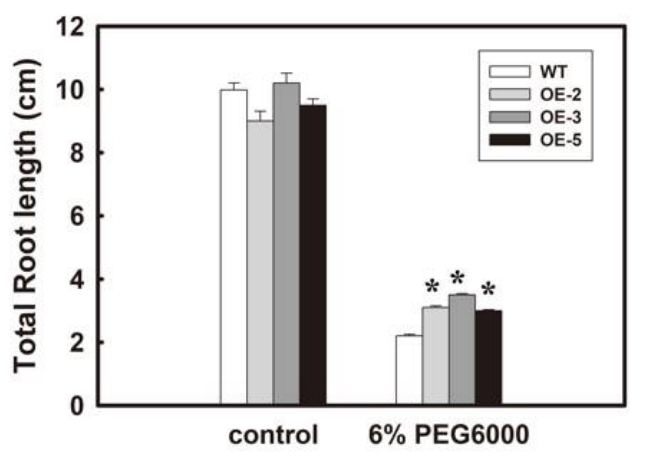

C

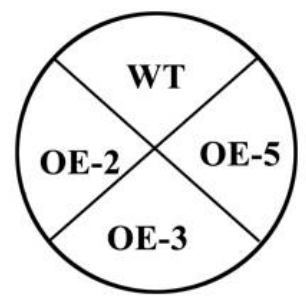

B

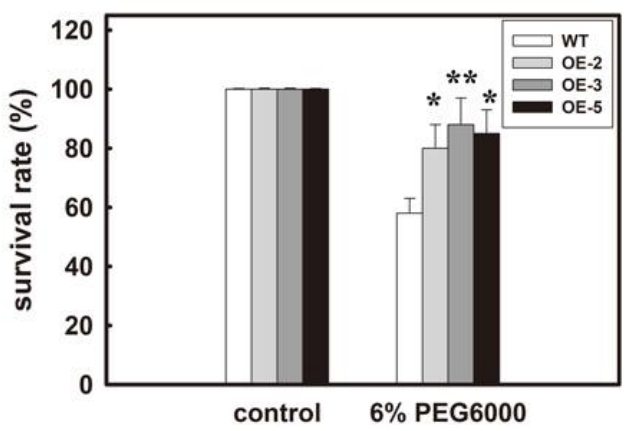

Drough treatment Re-watering
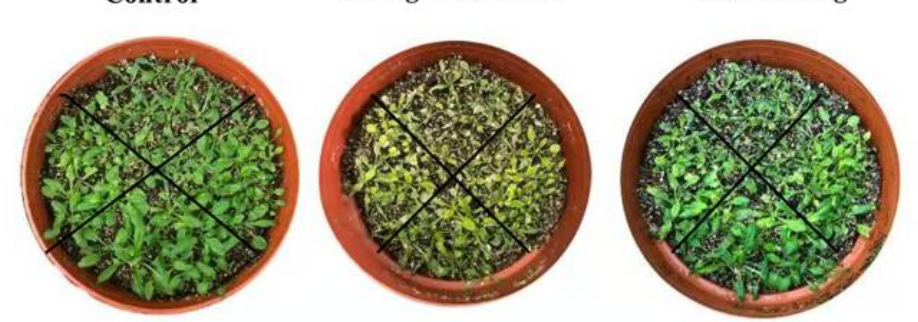

Figure 6. Improved drought tolerance in the transgenic Arabidopsis plants with the heterologous overexpression of GmTGA15. (A) Total root length of WT and transgenic lines grown in medium with and without (the control) $6 \%$ (m/v) PEG6000 for 8 days. (B) Survival rates after 1 week of recovery of the WT and transgenic Arabidopsis plants grown in soil under drought stress. (C) Experimental display of the spatial arrangements and the phenotypic observations of the WT and three lines of transgenic Arabidopsis plants (OE-2, OE-3, and OE-5). All values are presented as means of three independent replicates $(n=20)$, with the error bars indicating the standard deviations. The asterisks represent significant differences $\left({ }^{*} p<0.05\right.$ and $\left.{ }^{* *} p<0.01\right)$ compared with the controls.

\section{Discussion}

Studies have shown that the cis-elements in the promoter region of a gene are closely related to the biological function of the gene [35]. Our previous analysis of the cis-acting elements showed that the promoter region of GmTGA15 contained the pathogen-related ciselements such as ERE, W-box, P-box, or WUN-motif, indicating that GmTGA15 is involved in the defense of pathogens [24]. This observation is consistent with the involvement of $T G A$ genes in response to pathogen attacks [36]. Furthermore, we identified the cis-elements related to stresses in the promoter region of GmTGA15 in response to drought, salt, and hormones ABA, SA, GA, and MeJA. Therefore, it is speculated that GmTGA15 is involved in these responses.

It is well-known that environmental stresses such as salt, drought, high temperature, and oxidation affect the growth and development of plants, while TGA transcription factors participate in the response of plants to these stresses. Our results showed that GmTGA15 was induced to show varied expression patterns under different environmental stresses (i.e., drought and salt) and exogenous hormones (i.e., ABA, GA, SA, and MeJA), with the greatest response to drought, suggesting that GmTGA15 plays different roles under different abiotic stresses and hormones (Figure 1). Furthermore, our results of the qRT-PCR analysis showed that the expression levels in different tissues or organs during different developmental stages varied greatly. For example, roots showed the highest expression level among all examined organs during the four developmental stages, and were $\sim 68$-fold higher than those in either stems or leaves during the seedling stage (Figure 2A). These results were consistent with those reported recently [23], further suggesting the existence of the diverse tissue- or organ-specific regulatory mechanisms (Figure 2). Studies have extensively shown that plant bZIP transcription factors are induced and expressed by 
plant hormones such as SA, MeJA, ABA, and ethylene (ETH) [37-40]. Furthermore, it has been reported that different TGA transcription factors play different roles, showing varied specificities and responding differently to different adverse conditions. For example, Zhang et al. [41] showed that the wheat TabZIP1 gene is rapidly induced by exogenous MeJA and ETH, but not influenced by SA. Our results showed that exogenous SA and GA rapidly induce the expression of GmTGA15 at high levels, in contrast with the slow expression under the treatment of exogenous ABA and MeJA and indicating that GmTGA15 may participate in SA and GA signal pathways to regulate gene expression in response to the adverse stresses (Figure 1).

Studies have shown that drought generates osmotic stress, leading to an increase in the amount of reactive oxygen species (ROSs), lipid peroxidation, protein oxidation, nucleic acid damage, and the activation of programmed cell death, while transgenic plants demonstrate enhanced drought tolerance by eliminating reactive oxygen species [42]. In order to study the molecular mechanisms of enhanced drought tolerance by GmTGA15 in genetically modified soybean hairy roots, the plant expression vector pCAMBIA3301GmTGA15 was constructed and then transformed into soybean hairy roots by Agrobacterium rhizogenes-mediated methods. The GmTGA15 transgenic soybean hairy roots were treated under drought stress induced by $20 \%$ PEG6000, with the contents of malondialdehyde (MDA), proline, and chlorophyll evaluated (Figure 5). As an indicator of the destruction of ROSs under stress conditions, the contents of MDA in transgenic plants were significantly reduced under salt and osmotic stresses [43]. As an osmotic substance, the accumulation of a large amount of proline plays an important role in stabilizing the macromolecules and cell membranes in transgenic plants under salt and osmotic stresses $[44,45]$. Furthermore, as an important functional substance absorbing and transmitting light energy in plant photosynthesis, the level of chlorophyll content, which is changed by drought stress, directly affects the performance and yields of photosynthesis in plants [46]. For example, studies have shown that chlorophyll content was decreased significantly by an elevated degree of drought stress [47]. Our results showed that the contents of both proline and chlorophyll were increased, while the contents of malondialdehyde was decreased, in the soybean hairy roots with an overexpression of GmTGA15. These results indicate that GmTGA15 increases the drought tolerance of soybean hairy roots by elevating the contents of proline and chlorophyll and lowering the contents of malondialdehyde. These results further indicate that GmTGA15 is probably involved in the regulation of the synthesis of proline, chlorophyll, and MDA. In our study, enhanced drought tolerance by GmTGA15 was further confirmed by its heterologous overexpression in transgenic Arabidopsis plants. Specifically, transgenic Arabidopsis plants showed significantly longer root lengths and lower mortality under drought-stress conditions than those of WT plants. These results are consistent with those reported previously showing enhanced tolerance to drought stress in both transgenic Arabidopsis plants and soybean hairy roots by the transcription factor GmTGA17 [23]. We note that due to their shared molecular similarities and functions in enhancing drought tolerance in soybean and Arabidopsis plants, it is speculated that there might be a synergistic effect between GmTGA15 and GmTGA17. Further studies are necessary to verify this speculation.

\section{Conclusions}

Our investigation of the overexpression of the transcription factor GmTGA15 in response to drought stress in transgenic soybean hairy roots and Arabidopsis plants has demonstrated that both abiotic stresses (drought and saline) and hormone treatments (SA, ABA, MeJA, and GA) greatly induced the expression of GmTGA15 in soybean plants. Results showed that the contents of both chlorophyll and proline were significantly increased, while the content of malondialdehyde (MDA) was significantly decreased in the soybean hairy roots with the overexpression of GmTGA15 in comparison to wild type in response to drought stress. Furthermore, the transgenic Arabidopsis plants with an overexpression of GmTGA15 showed significantly advantageous phenotypes (i.e., longer roots and lower 
mortality) compared to that of the wild type under the simulated drought conditions, suggesting that GmTGA15 promotes tolerance to drought stress in both soybean and Arabidopsis plants. Due to the severe deterioration caused by the various environmental factors (e.g., drought and soil salinization), it is important to breed drought-resistance crops. This study provides the scientific evidence for further functional analysis of soybean TGA transcription factors in the drought stress and breeding of drought-resistance crops.

Supplementary Materials: The following are available online at https://www.mdpi.com/2073 $-4395 / 11 / 1 / 170 / s 1$, Figure S1: Construction of soybean plant expression vector pCAMBIA3301GmTGA15 (A) transformed into Agrobacterium rhizogenes (B). Lane M indicates the DL2000 markers. Lanes 1 and 2 in (A) indicate the PCR products (1089 bp) of GmTGA15 amplified from two vectors randomly chosen. Lanes 1-4 in (B) present the PCR products (1089 bp) of GmTGA15 amplified from four lines of randomly chosen vectors transformed into Agrobacterium rhizogenes.

Author Contributions: Conceptualization, Z.C. and X.C.; methodology, Z.C., X.Y., and X.C.; software, Y.Z. (Ying Zhou); validation, X.F., X.Y., and H.L.; formal analysis, Z.C. and X.C.; investigation, X.F., H.L., and Y.Z. (Yingying Zhang); resources, X.C.; data curation, X.F. and Y.Z. (Yingying Zhang); writing-original draft preparation, Z.C., Y.Z. (Ying Zhou) and X.C.; writing-review and editing, Y.Z. (Ying Zhou) and X.C.; visualization, Y.Z. (Yingying Zhang) and H.L.; supervision, Y.Z. (Ying Zhou) and X.C.; project administration, X.C.; funding acquisition, X.C. All authors have read and agreed to the published version of the manuscript.

Funding: This research was funded by the National Natural Science Foundation of China (31771868), the National Science Foundation of Jilin Province (20180101026), and the National College Students Innovation and Entrepreneurship Project in China (201610193047). The APC was funded by X.C.

Conflicts of Interest: The authors declare no conflict of interest.

\section{References}

1. Shinozaki, K.; Yamaguchi-Shinozaki, K.; Seki, M. Regulatory network of gene expression in the drought and cold stress responses. Curr. Opin. Plant Biol. 2003, 6, 410-417. [CrossRef]

2. Shinozaki, K.; Yamaguchi-Shinozaki, K. Gene networks involved in drought stress response and tolerance. J. Exp. Bot. 2007, 58, 221-227. [CrossRef]

3. Butterbrodt, T.; Thurow, C.; Gatz, C. Chromatin immunoprecipitation analysis of the tobaccoPR-1a- and the truncatedCaMV 35Spromoter reveals differences in salicylic acid-dependent TGA factor binding and histone acetylation. Plant Mol. Biol. 2006, 61, 665-674. [CrossRef] [PubMed]

4. Katagiri, F.; Lam, E.; Chua, N.H. Two tobacco DNA-binding proteins with homology to the nuclear factor CREB. Nature 1989, 340, 727-730. [CrossRef] [PubMed]

5. Miao, Z.H.; Liu, X.; Lam, E. TGA3 is a distinct member of the TGA family of bZIP transcription factors in Arabidopsis thaliana. Plant Mol. Biol. 1994, 25, 1-11. [CrossRef]

6. Chern, M.S.; Fitzgerald, H.A.; Yadav, R.C.; Canlas, P.E.; Dong, X.; Ronald, P.C. Evidence for a disease-resistance pathway in rice similar to the NPR1-mediated signaling pathway in Arabidopsis. Plant J. 2001, 27, 101-113. [CrossRef]

7. Zhou, J.M.; Trifa, Y.; Silva, H.; Pontier, D.; Lam, E.; Shah, J.; Klessig, D.F. NPR1 differentially interacts with members of the TGA/OBF family of transcription factors that bind an element of the PR-1 gene required for induction by salicylic acid. Mol. Plant Microbe Interact. 2000, 13, 191-202. [CrossRef]

8. Zhang, J.Y.; Qu, S.C.; Du, X.L.; Qiao, Y.S.; Cai, B.H.; Guo, Z.R.; Zhang, Z. Overexpression of the Malus hupehensis MhTGA2 gene, a novel bZIP transcription factor for increased tolerance to salt and osmotic stress in transgenic tobacco. Int. J. Plant Sci. 2012, 173, 441-453. [CrossRef]

9. Zhu, Q.G.; Wang, M.M.; Gong, Z.Y.; Fang, F.; Sun, N.J.; Li, X.; Grierson, D.; Yin, X.R.; Chen, K.S. Involvement of DkTGA1 transcription factor in anaerobic response leading to persimmon fruit postharvest de-astringency. PLoS ONE 2016, 11, e0155916. [CrossRef]

10. Espín, F.M.I.; Peraza-Echeverria, S.; Fuentes, G.; Santamaria, J.M. In silico cloning and characterization of the TGA (TGACG MOTIF-BINDING FACTOR) transcription factors subfamily in Carica papaya. Plant Physiol. Biochem. 2012, 54, 113-122. [CrossRef]

11. Gatz, C. From pioneers to team players: TGA transcription factors provide a molecular link between different stress pathways. Mol. Plant Microbe Interact. 2013, 26, 151-159. [CrossRef] [PubMed]

12. Huang, Q.; Wang, Y.; Li, B.; Chang, J.; Chen, M.; Li, K.; Yang, G.; He, G. TaNAC29, a NAC transcription factor from wheat, enhances salt and drought tolerance in transgenic Arabidopsis. BMC Plant Biol. 2018, 15, 268. [CrossRef] [PubMed]

13. Sun, T.; Busta, L.; Zhang, Q.; Ding, P.; Jetter, R.; Zhang, Y. TGACG-BINDING FACTOR 1 (TGA1) and TGA4 regulate salicylic acid and pipecolic acid biosynthesis by modulating the expression of SYSTEMIC ACQUIRED RESISTANCE DEFICIENT1 (SARD1) and CALMODULIN-BINDING PROTEIN 60g (CBP60g). New Phytol. 2017, 217, 344-354. [CrossRef] [PubMed] 
14. Alvarez, J.M.; Riveras, E.; Vidal, E.A.; Gras, D.E.; Contreras-Lopez, O.; Tamayo, K.P.; Aceituno, F.; Gomez, I.; Ruffel, S.; Lejay, L.; et al. Systems approach identifies TGA1 and TGA4 transcription factors as important regulatory components of the nitrate response of Arabidopsis thaliana roots. Plant J. 2015, 80, 1-13. [CrossRef]

15. Canales, J.; Contreras-Lopez, O.; Alvarez, J.M.; Gutierrez, R.A. Nitrate induction of root hair density is mediated by TGA1/TGA4 and CPC transcription factors in Arabidopsis thaliana. Plant J. 2017, 92, 305-316. [CrossRef] [PubMed]

16. Zhong, L.; Chen, D.; Min, D.; Li, W.; Xu, Z.; Zhou, Y.; Li, L.; Chen, M.; Ma, Y. AtTGA4, a bZIP transcription factor, confers drought resistance by enhancing nitrate transport and assimilation in Arabidopsis thaliana. Biochem. Biophys. Res. Commun. 2015, 457, 433-439. [CrossRef]

17. Wu, J.J. Functional Characterization of Arabidopsis Transcription Factor TGA7 in Plant Responses to Drought Stress. Ph.D. Thesis, China Agricultural University, Beijing, China, 2014.

18. Liao, Y.; Zou, H.F.; Wei, W.; Hao, Y.J.; Tian, A.G.; Huang, J.; Liu, Y.F.; Zhang, J.S.; Chen, S.Y. Soybean GmbZIP44, GmbZIP62 and GmbZIP78 genes function as negative regulator of ABA signaling and confer salt and freezing tolerance in transgenic Arabidopsis. Planta 2008, 228, 225-240. [CrossRef]

19. An, J.P.; Yao, J.F.; Xu, R.R.; You, C.X.; Wang, X.F.; Hao, Y.J. Apple bZIP transcription factor MdbZIP44 regulates ABA-promoted anthocyanin accumulation. Plant Cell Environ. 2018, 41, 2678-2692. [CrossRef]

20. Li, Y.Y.; Chen, Q.Z.; Nan, H.Y.; Li, X.; Lu, S.; Zhao, X.; Liu, B.; Guo, C.; Kong, F.; Cao, D. Overexpression of GmFDL19 enhances tolerance to drought and salt stresses in soybean. PLoS ONE 2017, 12, e0179554. [CrossRef]

21. Xu, Z.; Ali, Z.; Xu, L.; He, X.; Huang, Y.; Yi, J.; Shao, H.; Ma, H.; Zhang, D. The nuclear protein GmbZIP110 has transcription activation activity and plays important roles in the response to salinity stress in soybean. Sci. Rep. 2016, 6, 20366. [CrossRef]

22. Ullah, I.; Magdy, M.; Wang, L.; Liu, M.; Li, X. Genome-wide identification and evolutionary analysis of TGA transcription factors in soybean. Sci. Rep. 2010, 9, 11186. [CrossRef] [PubMed]

23. Li, B.; Liu, Y.; Cui, X.Y.; Fu, J.D.; Zhou, Y.B.; Zheng, W.J.; Lan, J.H.; Jin, L.G.; Chen, M.; Ma, Y.Z.; et al. Genome-wide characterization and expression analysis of soybean TGA transcription factors identified a novel TGA gene involved in drought and salt tolerance. Front. Plant Sci. 2019, 10, 549. [CrossRef] [PubMed]

24. Yuan, X.; Li, Y.; Jiang, Y.; Zhou, Y.; Chen, Z.; Cui, X. Molecular cloning, transcriptional activation and subcellular localization of GmTGA15 in soybean (Glycine max). J. Jilin Agric. Univ. 2021, in press.

25. Kereszt, A.; Li, D.X.; Indrasumunar, A.; Nguyen, C.D.T.; Nontachaiyapoom, S.; Kinkema, M.; Gresshoff, P.M. Agrobacterium rhizogenes-mediated transformation of soybean to study root biology. Nat. Protoc. 2007, 2, 948-952. [CrossRef] [PubMed]

26. Xiong, B.; Wang, Y.; Zhang, Y.; Ma, M.; Gao, Y.; Zhou, Z.; Wang, B.; Wang, T.; Lv, X.; Wang, X.; et al. Alleviation of drought stress and the physiological mechanisms in Citrus cultivar (Huangguogan) treated with methyl jasmonate. Biosci. Biotechnol. Biochem. 2020, 84, 1958-1965. [CrossRef]

27. Ullah, A.; Manghwar, H.; Shaban, M.; Khan, A.H.; Akbar, A.; Ali, U.; Ali, E.; Fahad, S. Phytohormones enhanced drought tolerance in plants: A coping strategy. Environ. Sci. Pollut. Res. Int. 2018, 25, 33103-33118. [CrossRef]

28. Livak, K.J.; Schmittgen, T.D. Analysis of relative gene expression data using real-time quantitative PCR and the 2(-Delta Delta C(T)) method. Methods 2001, 25, 402-408. [CrossRef]

29. El-Esawi, M.A.; Alayafi, A.A. Overexpression of rice Rab7 gene improves drought and heat tolerance and increase grain yield in rice. Genes 2019, 10, 56. [CrossRef]

30. Clough, S.J.; Bent, A.F. Floral dip: A simplified method for Agrobacterium-mediated transformation of Arabidopsis thaliana. Plant J. 1998, 16, 735-743. [CrossRef]

31. Bates, L.S.; Waldren, R.P.; Teare, I.D. Rapid determination of free proline for water-stress studies. Plant Soil 1973, 39, $205-207$. [CrossRef]

32. Cackmak, I.; Horst, W.J. Effect of Aluminum on lipid peroxisdation, superoxide dismutase, catalase, and peroxidase activities in root tips of soybean (Glycine max). Physiol. Plant. 1991, 83, 463-468. [CrossRef]

33. Arnon, D.I. Copper enzymes in isolated chloroplasts. Polyphenoloxidase in Beta vulgaris. Plant Physiol. 1949, 24, 1-15. [CrossRef] [PubMed]

34. Tang, Q.Y.; Zhang, C.X. Data processing system (DPS) software with experimental design, statistical analysis and data mining developed for use in entomological research. Insect Sci. 2012, 20, 254-260. [CrossRef] [PubMed]

35. Cheng, M.C.; Liao, P.M.; Kuo, W.W.; Lin, T.P. The Arabidopsis ETHYLENE RESPONSE FACTOR1 regulates abiotic stressresponsive gene expression by binding to different cis-acting elements in response to different stress signals. Plant Physiol. 2013, 162, 1566-1582. [CrossRef] [PubMed]

36. Wei, K.; Chen, J.; Wang, Y.; Chen, Y.; Chen, S.; Lin, Y.; Pan, S.; Zhong, X.; Xie, D. Genome-wide analysis of bZIP-encoding genes in maize. DNA Res. 2012, 19, 463-476. [CrossRef]

37. Lee, S.C.; Choi, H.W.; Hwang, I.S.; Choi, D.S.; Hwang, B.K. Functional roles of the pepper pathogen-induced bZIP transcription factor, CAbZIP1, in enhanced resistance to pathogen infection and environmental stresses. Planta 2006, 224, 1209-1225. [CrossRef]

38. Schlögl, P.S.; Nogueira, F.T.S.; Drummond, R.; Felix, J.M.; De Rosa, V.E.; Vicentini, R.; Leite, A.; Ulian, E.C.; Menossi, M Identification of new ABA- and MEJA-activated sugarcane bZIP genes by data mining in the SUCEST database. Plant Cell Rep. 2008, 27, 335-345. [CrossRef] 
39. Zhang, Y.; Zhang, G.; Xia, N.; Wang, X.J.; Huang, L.L.; Kang, Z.S. Cloning and characterization of a bZIP transcription factor gene in wheat and its expression in response to stripe rust pathogen infection and abiotic stresses. Physiol. Mol. Plant Pathol. 2008, 73, 88-94. [CrossRef]

40. Zou, M.; Guan, Y.; Ren, H.; Zhang, F.; Chen, F. A bZIP transcription factor, OsABI5, is involved in rice fertility and stress tolerance. Plant Mol. Biol. 2008, 66, 675-683. [CrossRef]

41. Zhang, Y.; Xu, S.; Ding, P.; Wang, D.; Cheng, Y.T.; He, J.; Gao, M.; Xu, F.; Li, Y.; Zhu, Z.; et al. Control of salicylic acid synthesis and systemic acquired resistance by two members of a plant-specific family of transcription factors. Proc. Natl. Acad. Sci. USA 2010, 107, 18220-18225. [CrossRef]

42. Noctor, F.G. Redox Homeostasis and Antioxidant Signaling: A metabolic interface between stress perception and physiological responses. Plant Cell 2005, 17, 1866-1875.

43. Sharma, P.; Jha, A.B.; Dubey, R.S.; Pessarakli, M. Reactive oxygen species, oxidative damage, and antioxidative defense mechanism in plants under stressful conditions. J. Bot. 2012, 2012, 1-26. [CrossRef]

44. Mahajan, S.; Tuteja, N. Cold, salinity and drought stresses: An overview. Arch. Biochem. Biophys. 2005, 444, 139-158. [CrossRef] [PubMed]

45. Cao, Y.J.; Wei, Q.; Liao, Y.; Song, H.L.; Li, X.; Xiang, C.B.; Kuai, B.K. Ectopic overexpression of AtHDG11 in tall fescue resulted in enhanced tolerance to drought and salt stress. Plant Cell Rep. 2009, 28, 579-588. [CrossRef]

46. Liu, X.; Li, L.; Li, M.; Su, L.; Lian, S.; Zhang, B.; Li, X.; Ge, K.; Li, L. AhGLK1 affects chlorophyll biosynthesis and photosynthesis in peanut leaves during recovery from drought. Sci. Rep. 2018, 8, 2250. [CrossRef]

47. Cui, J.; Jiang, N.; Zhou, X.; Hou, X.; Yang, G.; Meng, J.; Luan, Y. Tomato MYB49 enhances resistance to Phytophthora infestans and tolerance to water deficit and salt stress. Planta 2018, 248, 1487-1503. [CrossRef] 